Article

\title{
Convolutional Two-Stream Network Using Multi-Facial Feature Fusion for Driver Fatigue Detection
}

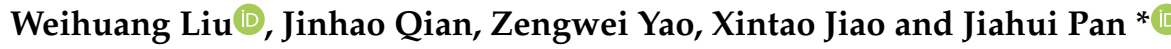 \\ School of Software, South China Normal University, Guangzhou 510641, China; 20152005073@m.scnu.edu.cn (W.L.); \\ 20152005078@m.scnu.edu.cn (J.Q.); 20152005086@m.scnu.edu.cn (Z.Y.); g_xtjiao@126.com (X.J.) \\ * Correspondence: panjiahui@m.scnu.edu.cn
}

Received: 23 February 2019; Accepted: 29 April 2019; Published: 14 May 2019

check for updates

\begin{abstract}
Road traffic accidents caused by fatigue driving are common causes of human casualties. In this paper, we present a driver fatigue detection algorithm using two-stream network models with multi-facial features. The algorithm consists of four parts: (1) Positioning mouth and eye with multi-task cascaded convolutional neural networks (MTCNNs). (2) Extracting the static features from a partial facial image. (3) Extracting the dynamic features from a partial facial optical flow. (4) Combining both static and dynamic features using a two-stream neural network to make the classification. The main contribution of this paper is the combination of a two-stream network and multi-facial features for driver fatigue detection. Two-stream networks can combine static and dynamic image information, while partial facial images as network inputs can focus on fatigue-related information, which brings better performance. Moreover, we applied gamma correction to enhance image contrast, which can help our method achieve better results, noted by an increased accuracy of $2 \%$ in night environments. Finally, an accuracy of $97.06 \%$ was achieved on the National Tsing Hua University Driver Drowsiness Detection (NTHU-DDD) dataset.
\end{abstract}

Keywords: fatigue detection; multi-task cascaded convolutional networks; optical flow; gamma correction; feature fusion

\section{Introduction}

According to the National Highway Traffic Safety Administration report, 22\% to 24\% of traffic accidents are caused by driver fatigue. Driver fatigue during driving can increase the risk of accidents by four to six times. Frequent occurrence of traffic accidents seriously threatens the safety of people's life and property. Therefore, the study of driver fatigue detection is of great significance.

Research shows that fatigue is closely related to psychophysiological changes, such as blink rate, heart rate, anxiety, etc. [1]. Nowadays, there are various techniques to measure driver fatigue. These techniques can be generally classified into three categories: vehicle-focused, driver-focused, and computer vision-based methods. Driver-focused methods focus on psychophysiological parameters such as using electroencephalogram (EEG) data [2-4], which would be an intrusive mechanism for detecting driver status. Vehicle-focused methods detect the running condition of the vehicle and the status of the steering wheel [5], which have specific limitation factors such as highway driving. Charlotte [6] combined vehicle-focused and driver-focused methods, measuring physiological and behavioral indicators to analyze and prevent accidents. Because of the rapid development of deep learning, driver fatigue detection has been an active research topic in the field of computer vision in recent years. In driver fatigue detection based on computer vision, some researchers focus on the driver's mouth movement $[7,8]$, while others study the relation between fatigue and eye movement [9-12]. Mandal et al. calculated the blink rate as a basis for judging driving [13]. Saradadevi \& Bajaj used support vector machines to classify normal and yawning mouths [14]. Ji et al. [15] combined multiple 
visual cues to get a more robust and accurate model, which included eyelid movement, gaze movement, head movement, and facial expression.

Although an EEG is highly correlated with the driver's mental state and is most sensitive to fatigue detection, it has a large amount of redundant information, which will affect the efficiency and accuracy of detection. In addition, it also requires drivers to wear related devices, which is an intrusive mechanism for driver. Driver fatigue detection based on the vehicle driving mode is greatly influenced by external factors and the driver's own driving habits; therefore, the detection accuracy is not so high. The driver fatigue detection method based on computer vision not only has high accuracy, but also has no impact on the driver's driving. Therefore, it is more applicable.

With the prevalence of convolutional neural networks (CNNs), more and more driver fatigue monitoring algorithms have used convolutional neural networks as underlying algorithms, and more and more variants have been invented. Park et al. [16] presented the Driver Drowsiness Detection (DDD) network. It integrated the results of three existing networks by support vector machine (SVM) to classify the categories of videos, which cannot monitor driver drowsiness online. Three-dimensional convolutional neural networks (3D-CNNs) were applied to extract spatial and temporal information by $\mathrm{Yu}$ et al. [17], but the approach took advantage of global face images, which did not have the flexibility to configure patches that contained most of the drowsiness information. Miguel [18] also used global facial information to detect driver fatigue. Celona [19] proposed a sequence-level classification model that was able to simultaneously estimate the status of the driver's eyes, mouth, head, and drowsiness. Long-term multi-granularity deep framework [20] combined long short-term memory network (LSTM) [21] and CNN with multi-granularity; however, this method did not contain dynamic information that behaved in the time dimension. In order to reduce computation, Rateb [22] used multi-layer perceptron instead of $\mathrm{CNN}$ to detect fatigue, which took face landmarks coordinates as the input; however, it lost facial information to some extent.

Some researchers, such as Liu [23] and Reddy [24], used partial facial images as data for fatigue monitoring without using global facial images. The reason for this was that partial facial features of the eyes and mouth contained action information, such as closing the eyes and yawning, which could be applied to fatigue monitoring. Instead of using global face area, we used local eye and mouth areas as our network input. According to Reddy [24], local face areas, such as the eyes and mouth, as a network input can reduce network training parameters and unnecessary noise effects. Thus, our proposed method used only the left eye and the mouth area. Another inspiration for our paper is from Simonyan [25], who proposed a two-stream ConvNet architecture. They achieved very good performance, in spite of limited training data, by incorporating spatial and temporal networks. Our paper also combined temporal stream and spatial stream information for the left eye and mouth.

\section{Methods}

In this paper, we proposed a method using multi-task cascaded convolutional neural networks (MTCNNs) to extract the mouth and the left eye area, and use gamma correction to enhance the image contrast. Combining optical flows of the mouth and the left eye regions, we used convolutional neural network $(\mathrm{CNN})$ to extract features for driver fatigue detection, which achieved good results on the National Tsing Hua University Driver Drowsiness Detection dataset (NTHU-DDD) [26]. Our algorithm flowchart is shown in Figure 1. 


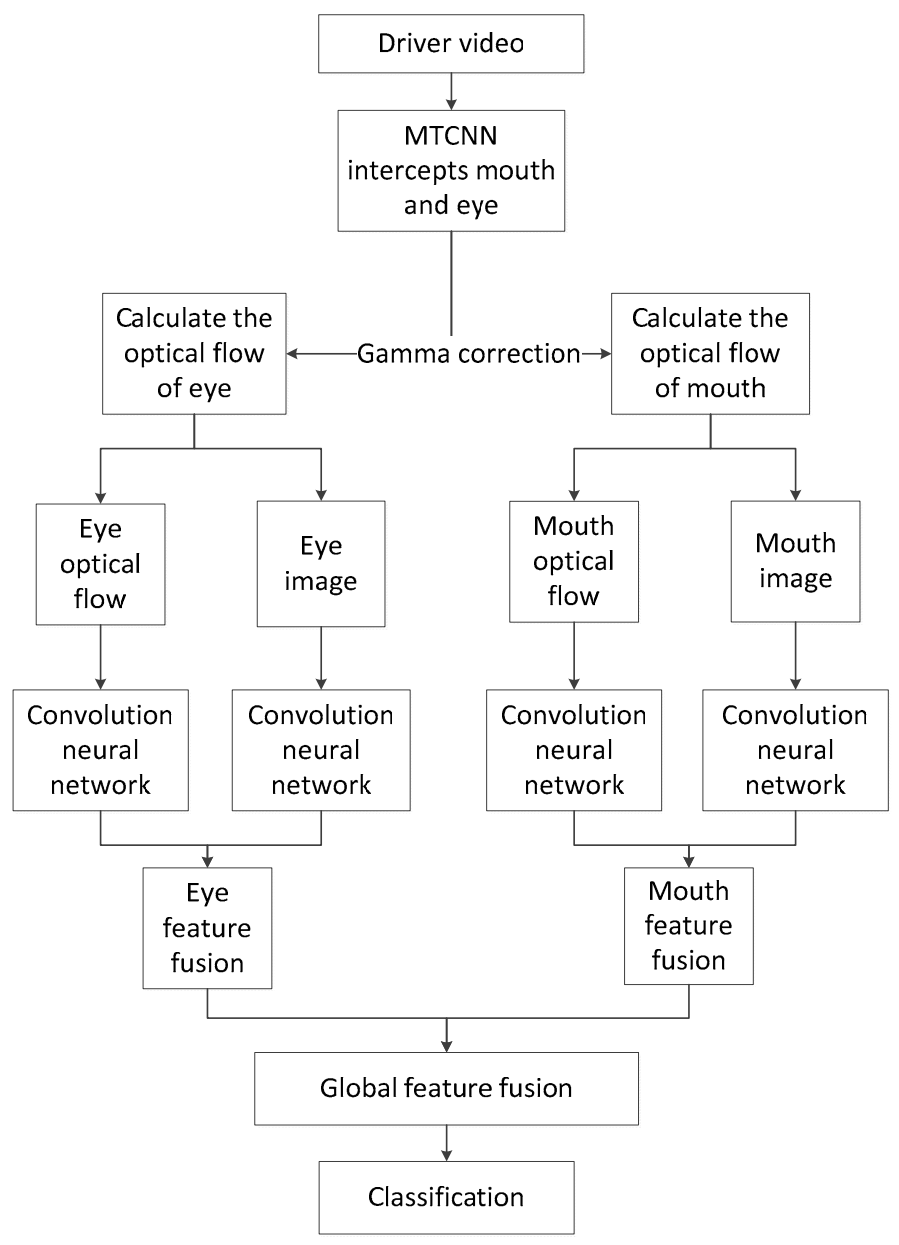

Figure 1. Algorithm flowchart for driver fatigue detection.

\subsection{Face Detection and Key Area Positioning}

Driver fatigue detection in real driving videos can be challenging because faces are affected by many factors such as the lighting conditions and the driver's gender, facial gestures, and facial expressions, etc. However, low-cost in-car cameras can only take low-resolution videos. Therefore, a high-performance face detector was needed. Even with a specific face area, positioning of the mouth and eye area was also very important, which contained important fatigue characteristics of the driver.

The Adaboost face detection algorithm [27], based on Haar features of the face, is not effective enough in a real, complex environment. It also cannot determine the eye area and mouth area. MTCNN [28] is known as one of the fastest and most accurate face detectors. With a cascading structure, MTCNN can jointly achieve rapid face detection and alignment. As a result of face detection and alignment, MTCNN obtained the facial bounding box and facial landmarks. In this paper, we used MTCNN for face detection and face alignment tasks.

MTCNN consists of three network architectures (P-Net, R-Net, and O-Net). In order to achieve scale invariance, the given image was scaled to different scales to form an image pyramid. In the first stage, shallow CNNs quickly generated candidate windows; in the second stage, more complex CNNs filtered candidate windows and discarded a large number of overlapping windows; in the third stage, more powerful CNNs were used to decide whether the candidate window should be discarded, while displaying five facial key positionings.

Proposal Network (P-Net) (shown in Figure 2): The main function of this network structure was to obtain the regression vector of the candidate window and bounding box in the face area. At the same time, it used the bounding box to do the regression and calibrate the candidate window, and then it merged the highly overlapping candidate boxes by non-maximum suppression (NMS). 
All input samples were first resized into $12 \times 12 \times 3$, and finally the P-Net output was obtained by a 1 $\times 1$ convolution kernel of three different output channels. P-Net output was divided into three parts: (1) face classification - the probability that the input image was a face; (2) bounding box-the position of the rectangle; and (3) facial landmark localization — the five key points of the input face sample.

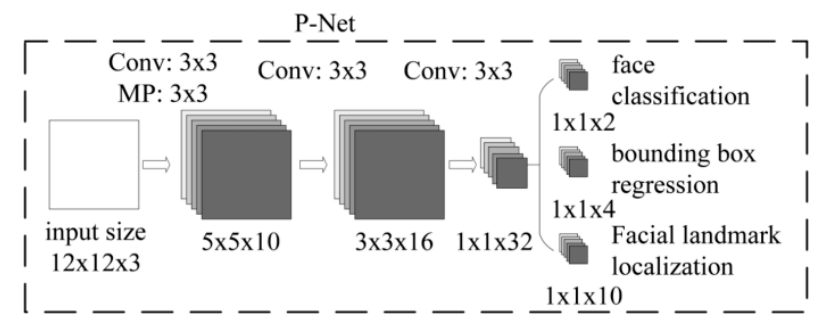

Figure 2. Proposal network (P-Net) structure.

Refine Network (R-Net) (shown in Figure 3): This network structure also removed the false positive region through bounding box regression and non-maximum suppression. However, since the network structure had one more fully connected layer than the P-Net network structure, a better effect of suppressing false positives could be obtained. All input samples were first resized to $24 \times 24 \times 3$, and finally the R-Net output was obtained by the fully connected layer. R-Net output was divided into three parts: (1) face classification - the probability that the input image was a face; (2) bounding box-the position of the rectangle; and (3) facial landmark localization-the five key points of the input face sample.

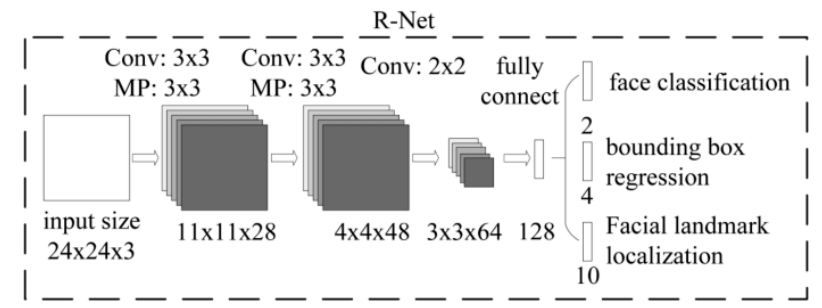

Figure 3. Refine Network (R-Net) structure.

Output Network (O-Net) (shown in Figure 4): This network structure had one more convolutional layer than R-Net, so the result of the processing was finer. The network worked similarly to R-Net, but it supervised the face area and obtained five coordinates representing the left eye, right eye, nose, left part of lip, and right part of lip. All input samples were first resized to $48 \times 48 \times 3$ dimensions, and finally the O-Net output was obtained by the fully connected layer. O-Net output was divided into three parts: (1) face classification - the probability that the input image was a face; (2) bounding box - the position of the rectangle; and (3) facial landmark localization-the five key points of the input face sample. All three networks set a threshold that represented the degree of overlap of face candidate windows in non-maximal suppression.

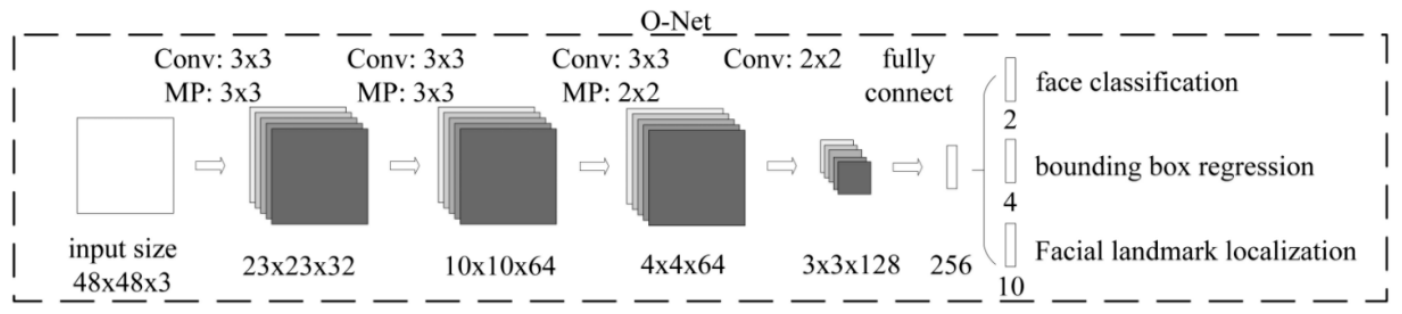

Figure 4. Output Network (O-Net) structure. 
Compared with common detection methods such as region-based convolutional neural networks (R-CNNs) [29], MTCNN is more suitable for face detection and is greatly improved in terms of speed and accuracy.

It was not adequate to determine the coordinates of the key point, thus, we needed to determine the eye and mouth area. In yawning, blinking, and other movements, mouth and eye sizes will change within a certain range. Therefore, in this paper, we used the eye coordinates as the center and the distance between the left and right part of lip as the length to determine a rectangular box for the eye area. Then, we used the midpoint between the left part of lip and the right part of lip as the center and the distance between the left part of lip and the right part of lip as the length to define a rectangular box for the mouth area. The actual effect is shown in Figure 5.

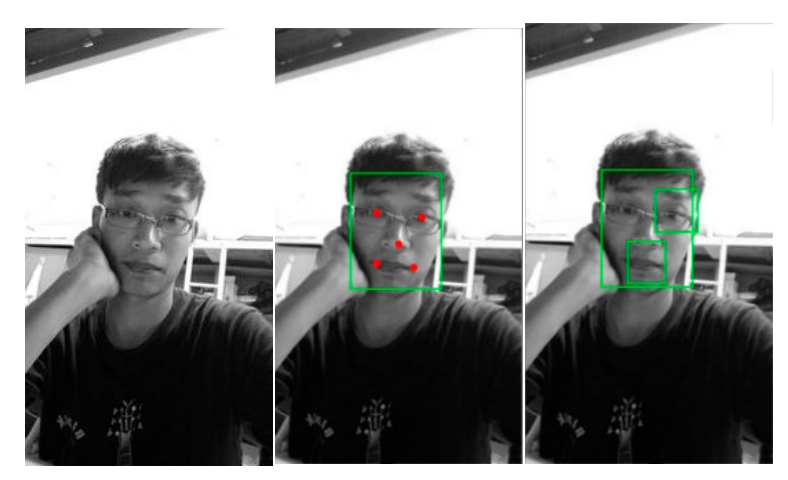

Figure 5. Multi-task cascaded convolutional neural network (MTCNN) detects face and key points.

\subsection{Gamma Correction}

In actual scene image acquisition, the image may be overexposed or underexposed due to environmental factors such as light exposure, resulting in non-uniform gray-level distribution. This will deteriorate the image quality and negatively affect the result of the calculation.

In digital image processing, gamma correction [30] is usually applied to the correction of the output image of the display device. Since Cathode Ray Tube (CRT), Light Emitting Diode (LED), and other display devices do not work in a linear manner when displaying colors, color output in the program will eventually have diminished brightness when outputted to the monitor. This phenomenon can affect the quality of the image when calculating lighting and real-time rendering, so the output image needs gamma correction. The gray value of the input image was non-linearly transformed by gamma correction. It improved the image contrast through detecting the dark part and the light part in the image signal and increased the ratio between these two parts.

In order to utilize the gray information of the image more effectively, we gamma-corrected the input image to reduce the influence of the non-uniform gray-level distribution of the image. Since the eye and the mouth captured from image through the MTCNN were a small area with almost no partial overexposure or partial underexposure, it was possible to subject the entire obtained eye and mouth image to gamma correction, which improved image contrast.

According to the gamma correction formula, the corresponding relationship between the input pixel $P_{\text {in }}$, the output pixel $P_{\text {out }}$, the gamma coefficient gamma, and the gray level scale is:

$$
P_{\text {out }}=\left(\frac{p_{\text {in }}}{\text { scale }}\right)^{\text {gamma }} \times \text { scale }
$$

It can be deduced from (1) that, given the input pixel $P_{i n}$, the expected output pixel $P_{\text {hope }}$, the gray level scale, we can find:

$$
\text { gamma }=\log _{\frac{P_{\text {hope }}}{\text { scale }}} \frac{P_{\text {in }}}{\text { scale }}
$$


Formula (2) was relative to a single pixel. When faced with an image, a single pixel of the above formula was replaced by a grayscale mean of one image. The relationship between the gray mean $P_{\text {mean }}$ and the number of pixels $n$ is:

$$
\left(P_{\text {mean }}\right)^{\text {gamma }} \approx \frac{\sum_{i}^{n}\left(P_{i}\right)^{\text {gamma }}}{n}
$$

Since it was almost impossible for every pixel of an image to be equal, the above formula should be an approximately equal sign.

As shown in Figure 6, through gamma correction, the gray value of the different input images were transformed into a roughly similar desired gray value given by us.

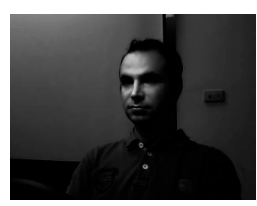

(a)

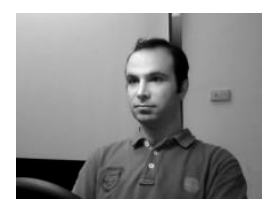

(b)

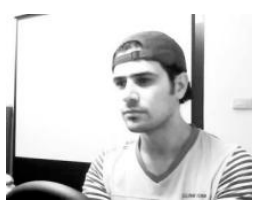

(c)

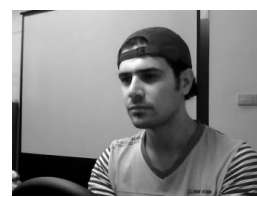

(d)

Figure 6. Original and gamma correction renderings. (a) Underexposed image. (b) Underexposed image after gamma correction. (c) Overexposed image. (d) Overexposed image after gamma correction.

\subsection{Optical Flow Calculation}

In real, three-dimensional space, the physical concept that describes the state of motion of an object is motion field. In the space of computer vision, the signal received by the computer is often two-dimensional image information. Because one dimension of information was lacking, it was no longer suitable for us to use motion fields to describe motion state. The optical flow field was used to describe the movement of three-dimensional space objects in the two-dimensional image, reflecting the motion vector field pixel.

As indicators of driver fatigue, yawning, blinking, etc. are not a static state but a dynamic action. Therefore, just a static image was not enough. By utilizing the change of pixels over time in the image sequence and the correlation between adjacent frames, optical flow was determined based on the corresponding relationship between the previous frame and the current frame, and it contained the dynamic information between adjacent frames. Unlike the method of using continuous frames for action recognition, such as LSTM and 3D-CNN, we used the dynamic information contained in optical flow data to replace the dynamic information provided by successive frames. In this paper, we fused the features of static and dynamic information to make driver fatigue detection better than using only static images.

Optical flow is observed in the imaging plane, and it is the instantaneous velocity of the pixel motion of an object moving through space. It uses the change in pixels over time in the image sequence and the correlation between adjacent frames to find the corresponding relationship between the previous frame and the current frame, then it calculates the motion information of objects between adjacent frames. Suppose there is a vector set $(x, y, t)$ in every moment, which represents the instantaneous velocity of the specified coordinate $(x, y)$ at the moment of $t$. Let $I(x, y, t)$ represent the pixel brightness of point $(x, y)$ at the moment of $t$, and in a very short period of time $\Delta t,(x, y)$ increases $(\Delta x, \Delta y)$ respectively, thus, we can get:

$$
\begin{aligned}
& I(x+\Delta x, y+\Delta y, t+\Delta t)= \\
& I(x, y, t)+\partial I / \partial x \Delta x+\partial I / \partial y \Delta y+\partial I / \partial t \Delta t
\end{aligned}
$$


At the same time, taking into account that the displacement of two adjacent frames is short enough, represented by:

$$
I(x, y, t)=I(x+\Delta x, y+\Delta y, t+\Delta t)
$$

we get:

$$
\begin{gathered}
\partial I / \partial x \Delta x+\partial I / \partial y \Delta y+\partial I / \partial t \Delta t=0 \\
\partial I / \partial x \Delta x / \Delta t+\partial I / \partial y \Delta y / \Delta t+\partial I / \partial t \Delta t / \Delta t=0
\end{gathered}
$$

Since:

$$
\Delta x / \Delta t=v_{x}, \Delta y / \Delta t=v_{y}
$$

The final conclusion can be drawn as:

$$
\partial I / \partial x v_{x}+\partial I / \partial y v_{y}+\partial I / \partial t=0
$$

where $v_{x}, v_{y}$ is the speed of $x, y$ respectively, which is called the optical flow of $I(x, y, t)$.

The Farneback algorithm [31] is a method of calculating dense optical flow. First of all, it approximates each neighborhood of two frames with a second-degree polynomial, which can be done efficiently with polynomial expansion transformations. Next, by observing how an exact polynomial transforms under translation, a method of estimating the optical flow is derived from the polynomial expansion coefficients. With this dense optical flow, image registration at the pixel level is possible. Consequently, the effect of registration is significantly better than that of sparse optical flow registration.

During car driving, noting that the camera is fixed in the car, the driver's face optical flow is generated by the driver's face movement in the scene. In order to reflect the driver's dynamic face changes, we used the Farneback algorithm to calculate the dense optical flow between adjacent frames of left eye and mouth respectively, which is shown in Figure 7.

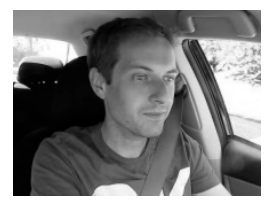

(a)

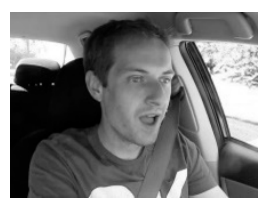

(b)

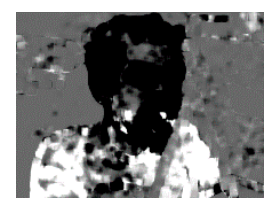

(c)

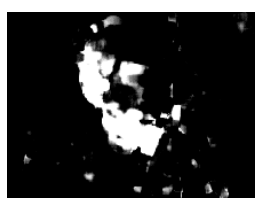

(d)

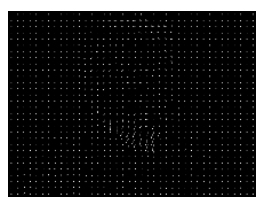

(e)

Figure 7. Farneback algorithm related images. (a) and (b): A pair of adjacent frames. (c) Horizontal component of the displacement vector field. (d) Vertical component of the displacement vector field.

(e) A close-up of dense optical flow for the adjacent frames.

\subsection{Fatigue Detection}

$\mathrm{CNN}$, which avoids complicated pre-processing of the image, can extract features with its special structure of local connection and weight sharing by directly inputting the original image. It has unique advantages in image processing.

Videos can be decomposed into spatial and temporal parts. The spatial part refers to appearance information of the independent frame, and the temporal part refers to motion information between two frames. The network structure proposed by reference [31] consisted of two deep networks, which handled the dimensionality of time and space separately. The video frame was sent to the first $\mathrm{CNN}$ to extract static features; meanwhile, the optical flow extracted from the video was sent to another CNN to extract dynamic features. Finally, the scores from the softmax layers of both networks were merged.

As a result of the natural state, the motion states of the left and right eyes of a person were consistent. Reddy et al. [24] proposed a method of driver drowsiness detection through inputting only the mouth region and the left eye region of a human face into the network. Compared with face inputs, this algorithm not only simplified the input but also achieved better results. 
Our algorithm first performed face detection of the driver. Then, the left eye area and the mouth area were intercepted into the fatigue detection network, combined with the optical flow image of the left eye and mouth, and the driver was judged whether they were in a normal, speaking, yawning, or dozing state. Unlike using LSTM and 3D-CNN to capture motion sequences from video frames to classify action, we used CNN to extract static features from the original image and dynamic features from the optical flow, thereby classifying a short time action.

The fatigue detection network, as shown in Figure 8, included four subnetworks. Input images in each sub-network were first resized to $50 \times 50 \times 3$ dimensions. The first subnetwork was to extract the feature of optical flow of the left eye. The second sub-network was to extract the feature of the left eye. The third sub-network was to extract the feature of optical flow of the mouth. The fourth sub-network was to extract the feature of the mouth. Together with the mouth and eye areas obtained after detection and interception, the calculation results of the optical flow of the mouth and eye areas were respectively inputted into the four subnetworks. After several layers of convolution and pooling, the left eye subnetwork and the left eye optical flow subnetwork were first fused to obtain further left eye regional features, while the mouth subnetwork and the mouth optical flow subnetwork were merged to obtain a further mouth regional feature. For the sake of obtaining global region characteristics, we merged the two new subnetworks and reintegrated them into the full connection layer. Finally, we inputted the data into the softmax layer for classification and obtained a $1 \times 5$ vector, which represented the probability of each class. To avoid over-fitting, an L2-regularization was added at each convolutional layer. At the same time, a dropout hyperparameter was added at each fully connected layer.

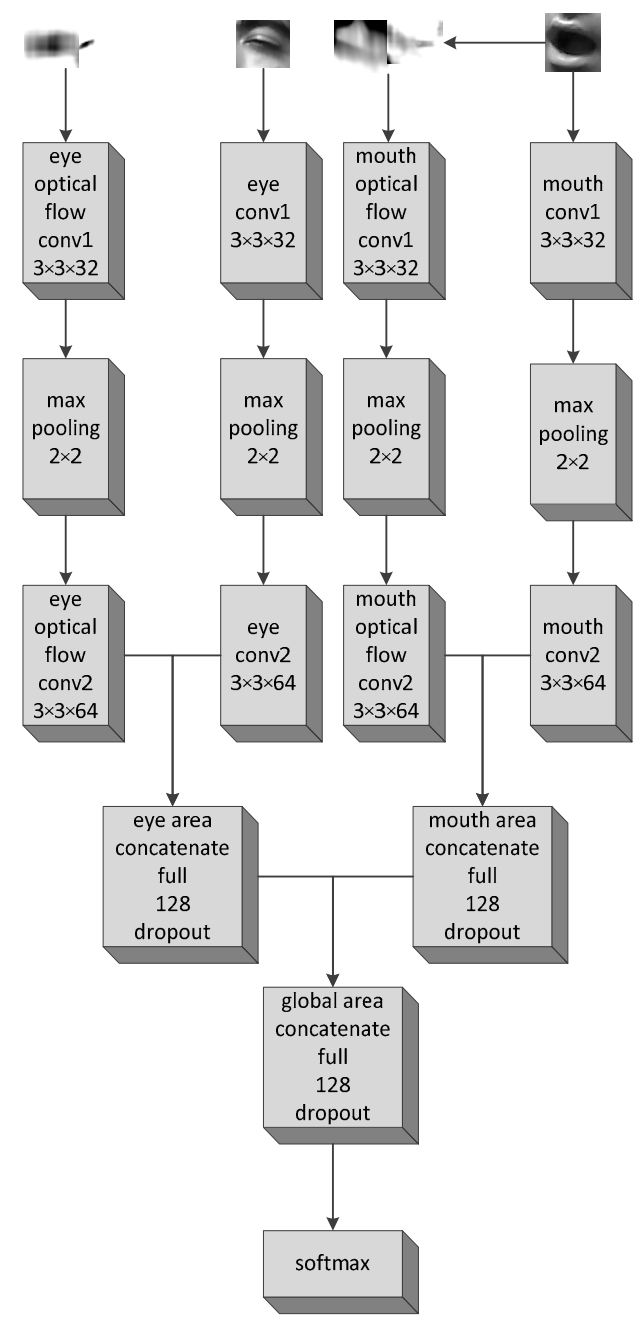

Figure 8. Fatigue detection network. 


\section{Experimental Results}

In the following, we provide competitive experimental results on the dataset used for driver drowsiness detection and compare the performance of state-of-the-art methods.

\subsection{National Tsing Hua University-Driver Drowsiness Detection (NTHU-DDD) Dataset}

The NTHU-DDD dataset [25] was a dataset developed by National Tsing Hua University, which was used at the Asian Conference on Computer Vision Workshop on Driver Drowsiness Detection. The entire dataset contained 36 subjects of different ethnicities, which were recorded with and without wearing glasses/sunglasses under a variety of daytime and nighttime simulated driving conditions. All movements of the driver were captured, including normal driving, yawning, slow blink rate, falling asleep, laughing, etc. The training set contained 360 video clips of 18 subjects, while the evaluation set consisted of 20 video clips of four subjects. The dataset contained a lot of normal, drowsy, talking, and yawn face data in various scenarios. Thus, the algorithm should consider robustness in all circumstances. Some screenshots from the dataset are shown in Figure 9.

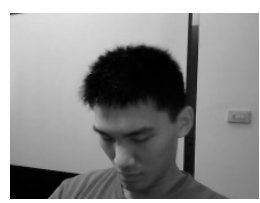

(a)

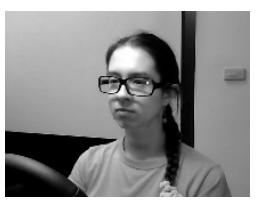

(b)

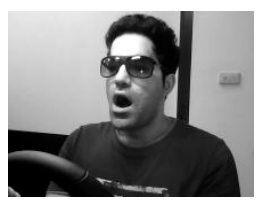

(c)

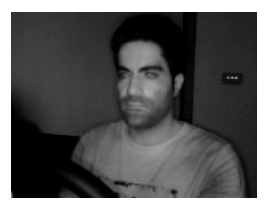

(d)

Figure 9. Sample image from the National Tsing Hua University-Driver Drowsiness Detection (NTHU-DDD) dataset. (a) Bareface (nodding); (b) wearing glasses (normal); (c) wearing sunglasses (yawning); and (d) bareface at night (normal).

\subsection{Experiment}

We trained our models using a training dataset with a stratified five-fold cross-validation [32], where data folds were chosen such that each fold had nearly the same class distribution as the original dataset, and it used an evaluation dataset for the test. Images were extracted one frame from every three frames in the videos, and they were labeled into five driver states: normal, drowsiness, nodding, talking, and yawning; the distribution of these classes in the dataset was around 5:9:2:5:3. Input images were first resized to a $50 \times 50$ size. The model input of the experiment without gamma correction was the original image, while the model with gamma correction input images had an average gray value of 120. We developed our models using the Keras framework and run experiments on GTX 1080 Ti. All of the layer weights were randomly initialized. We chose the hyper-parameters using a grid search. The network was trained using a batch gradient descent with a batch size of 128 and a dropout rate of 0.2. An initial learning rate of 0.1 was used in the optimization function Adadelta. Training was stopped when the validation loss did not improve for 50 iterations. The model was trained for around 230 iterations. The results are shown in Tables 1 and 2.

We tested the model at four different levels: (1) different scenarios; (2) different driving states; (3) different derived models; (4) average performance. Performances of the models without gamma correction, fatigue detection network (FDN), and with gamma correction, gamma fatigue detection network (GFDN), were comparable to state-of-the-art methods. In Table 1, we showed a comparison with state-of-the-art methods. Our models outperformed the existing methods in all of the scenarios, and the average performance surpassed all the state-of-the-art methods, which achieved a $97.06 \%$ accuracy. In addition, GFDN increased accuracy by $2 \%$ compared to FDN in a night environment.

We showed another result in Table 2. We obtained features before the softmax layer in the GFDN model as inputs, and we tested them with other classifiers. We chose and tuned four classification algorithms including k-nearest neighbors (KNNs) [33], centroid displacement-based k-nearest neighbors (CDNNs) [34], support vector machine (SVM) [35], and random forest (RF) [36]. Parameter k was 
tuned and chosen to be five and three in $\mathrm{KNN}$ and CDNN, respectively, through cross-validation. It was shown below that the accuracy of each derived model dropped slightly.

Considering the problem of unbalanced data, we added an F1-score to evaluate metrics. Table 3 shows the details in predicting different states using the GFDN model. According to Table 3 we could obtain the precision rate and the recall rate, which are shown in Table 4. Based on this, the F1-score was calculated to be 0.9688 .

Table 1. Drowsiness detection accuracies (\%) for different scenarios of the NTHU-DDD dataset.

\begin{tabular}{ccccccc}
\hline Scenarios & 3D-DCNN [28] & 3-nets DDD [29] & MLP [27] & seqMT-DMF [30] & $\begin{array}{c}\text { Fatigue Detection } \\
\text { Network (FDN) }\end{array}$ & $\begin{array}{c}\text { Gamma Fatigue Detection } \\
\text { Network (GFDN) }\end{array}$ \\
\hline Bareface & 75.10 & 69.83 & 87.12 & 84.46 & 97.18 & 97.36 \\
\hline Glasses & 72.30 & 75.93 & 84.85 & 77.35 & 97.60 & 97.35 \\
\hline Sunglasses & 70.90 & 69.86 & 75.11 & 86.43 & 97.13 & 97.86 \\
\hline Night-bareface & 68.40 & 74.93 & 81.40 & 82.48 & 94.03 & 96.25 \\
\hline Night-glasses & 68.30 & 74.77 & 76.15 & 87.18 & 94.26 & 96.14 \\
\hline Average & 71.20 & 73.06 & 80.93 & 83.44 & 96.60 & 97.06 \\
\hline
\end{tabular}

Table 2. Drowsiness detection accuracies (\%) for different states of the NTHU-DDD dataset in GFDN and derived models.

\begin{tabular}{cccccc}
\hline State & $\begin{array}{c}\text { GFDN-K-Nearest } \\
\text { Neighbor } \\
\text { (KNN) }\end{array}$ & $\begin{array}{c}\text { GFDN-Centroid } \\
\text { Displacement-Based K-Nearest } \\
\text { Neighbor (CDNN) }\end{array}$ & $\begin{array}{c}\text { GFDN-Support } \\
\text { Vector Machine } \\
\text { (SVM) }\end{array}$ & $\begin{array}{c}\text { GFDN-Random } \\
\text { Forest (RF) }\end{array}$ & GFDN \\
\hline normal & 92.86 & 93.42 & 93.96 & 94.61 & 93.70 \\
\hline drowsiness & 98.02 & 97.93 & 98.05 & 97.49 & 98.11 \\
\hline nodding & 96.00 & 96.11 & 96.51 & 96.21 & 96.31 \\
\hline talking & 97.37 & 97.02 & 97.18 & 96.83 & 97.75 \\
\hline yawning & 97.19 & 97.21 & 97.44 & 97.25 & 97.84 \\
\hline Average & 96.67 & 96.78 & 96.98 & 96.70 & 97.06 \\
\hline
\end{tabular}

Table 3. Drowsiness detection details for different states of the NTHU-DDD dataset in GFDN.

\begin{tabular}{cccccc}
\hline \multirow{2}{*}{ Real } & Normal & Drowsiness & Nodding & Talking & Yawning \\
\cline { 2 - 6 } & 9643 & 269 & 24 & 331 & 24 \\
\hline normal & 164 & 21,234 & 112 & 12 & 121 \\
\hline drowsiness & 11 & 183 & 5833 & 23 & 6 \\
\hline nodding & 204 & 9 & 39 & 12,614 & 38 \\
\hline talking & 15 & 167 & 19 & 19 & 9970 \\
\hline yawning & & & & &
\end{tabular}

Table 4. Precision rate and recall rate (\%) for different states.

\begin{tabular}{cccccc}
\hline & Normal & Drowsiness & Nodding & Talking & Yawning \\
\hline Precision Rate & 96.07 & 97.12 & 96.78 & 97.03 & 98.13 \\
\hline Recall rate & 93.70 & 98.11 & 96.31 & 97.75 & 97.84 \\
\hline
\end{tabular}

\section{Discussion}

In this paper, we employed two-stream networks, multi-facial features, and gamma correction for driver fatigue detection. Gamma corrections in the input image, input partial facial features, and fused dynamic information resulted in more accurate driver fatigue detection compared to existing methods. The experiment results showed that our GFDN model had an average accuracy of $97.06 \%$ on the NTHU-DDD dataset. 
It is not suitable to directly input the entire image, which contains extraneous information. The driver's face is only about $200 \times 150$ pixels, compared with the actual $640 \times 480$ image in the camera shot. The full image contains useless information and has a negative effect on classification. Furthermore, after the image is resized and sent to $\mathrm{CNN}$, it carries much less correlative information, which makes it difficult to learn features. Inputting partial facial images can avoid inaccurate classifications. Therefore, inputting the left eye and mouth area, which are closely related to driver fatigue, is a sensible idea.

Using only static images is not accurate enough for driver drowsiness detection; this is an action recognition task instead of an image recognition task. For instance, people are opening their mouths when both yawning and speaking. There is no difference in static images, while optical flow can reflect the difference. When the mouth and left eye are used as the inputs of the network, dynamic information between the continuous frames is not used. Better results can be obtained using a two-stream neural network that contains dynamic information in optical flow.

Poor image quality caused by non-uniform gray-level distribution can have negative effects on calculation results, for example, images taken in too bright or too dark environments. In experiments, input images are subjected to gamma correction to effectively improve the accuracy, which reduces the calculation errors caused by insufficient image contrast. It brings a $2 \%$ improvement in classification accuracy in night environments.

It was shown in Tables 2 and 3 that the normal state had the worst classification accuracy among all states, and most misclassified normal images were labeled into drowsiness and talking. We further checked the dataset and found that: (1) images for the whole duration of talking were labeled as talking, as shown in Figure 10, including images that looked like the normal state; (2) some drowsiness images looked like the normal state, except minor differences in eye sleepiness, as shown in Figure 11. These increase the difficulty of model classification.
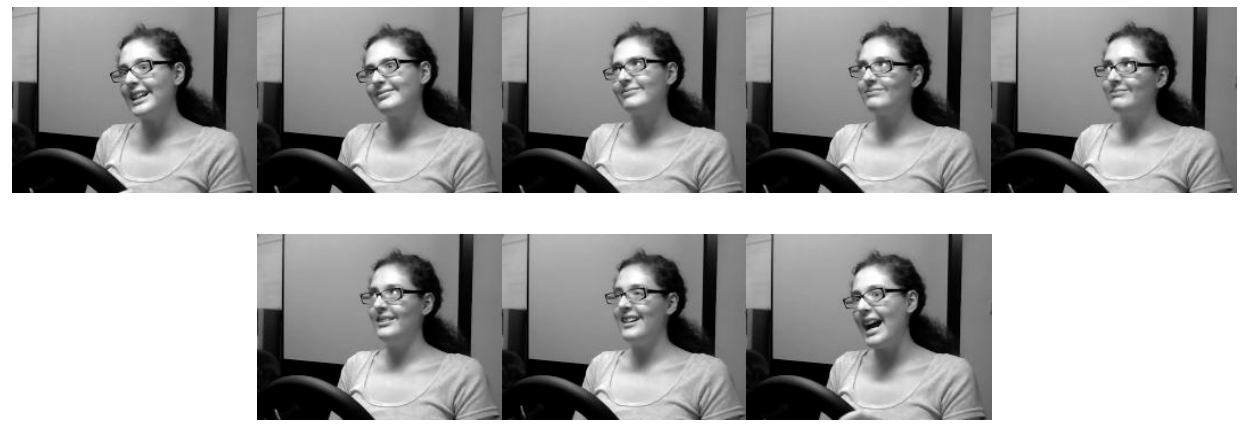

Figure 10. A video clip labeled as talking.

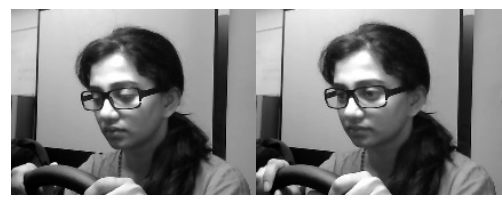

Figure 11. Two images labeled as drowsiness (left) and normal (right).

\section{Conclusions}

We proposed a driver fatigue detection algorithm based on multi-facial feature fusion, which not only avoided peripheral equipment on the driver's body, but also had high accuracy. We applied CNN and optical flow to video comprehension. We focused on partial information of the face, which was closely related to driver fatigue in the algorithm. We utilized optical flow to obtain dynamic information, and we used gamma correction to enhance image contrast. Thus, we achieved a competitive accuracy. From the experimental results, we can see that our model outperforms state-of-the-art methods. 
Author Contributions: Conceptualization, W.L.; methodology, W.L.; investigation, W.L. and J.Q.; experiment, W.L. and Z.Y.; writing-original draft preparation, W.L. and J.Q.; writing-review and editing, J.P.; supervision, J.P. and X.J.

Funding: This study was supported by the National Natural Science Foundation of China under Grants 61876067 and 61503143, the Guangdong Natural Science Foundation under Grant 2014A030310244 and the Pearl River S\&T Nova Program of Guangzhou under Grant 201710010038.

Conflicts of Interest: The authors declare no conflict of interest.

\section{References}

1. Lal, S.K.L.; Craig, A. Driver fatigue: Electroencephalography and psychological assessment. Psychophysiology 2002, 39, 313-321. [CrossRef] [PubMed]

2. Lal, S.K.; Craig, A.; Boord, P.; Kirkup, L.; Nguyen, H. Development of an algorithm for an EEG-based driver fatigue countermeasure. J. Saf. Res. 2003, 34, 321-328. [CrossRef]

3. Karuppusamy, N.S.; Kang, B.Y. Driver Fatigue Prediction Using EEG for Autonomous Vehicle. Adv. Sci. Lett. 2017, 23, 9561-9564. [CrossRef]

4. Min, J.; Wang, P.; Hu, J. Driver fatigue detection through multiple entropy fusion analysis in an EEG-based system. PLoS ONE 2017, 12, e0188756. [CrossRef] [PubMed]

5. Krajewski, J.; Sommer, D.; Trutschel, U.; Edwards, D.; Golz, M. Steering Wheel Behavior Based Estimation of Fatigue. In Proceedings of the Fifth International Driving Symposium on Human Factors in Driver Assessment Training \& Vehicle Design, Montana, IA, USA, 22-25 June 2009.

6. De Naurois, C.J.; Bourdin, C.; Stratulat, A.; Diaz, E.; Vercher, J.L. Detection and prediction of driver drowsiness using artificial neural network models. Accid. Anal. Prev. 2019, 126, 95-104. [CrossRef] [PubMed]

7. Fan, X.; Yin, B.C.; Sun, Y.F. Yawning Detection for Monitoring Driver Fatigue. In Proceedings of the 2007 International Conference on Machine Learning and Cybernetics, Hong Kong, China, 19-22 August 2007.

8. Rongben, W.; Lie, G.; Bingliang, T.; Lisheng, J. Monitoring Mouth Movement for Driver Fatigue or Distraction with One Camera. In Proceedings of the 7th International IEEE Conference on Intelligent Transportation Systems, Washington, WA, USA, 3-6 October 2004.

9. Singh, S.; Papanikolopoulos, N.P. Monitoring driver fatigue using facial analysis techniques. In Proceedings of the IEEE/IEEJ/JSAI International Conference on Intelligent Transportation Systems, Tokyo, Japan, 5-8 October 1999.

10. Devi, M.S.; Bajaj, P.R. Driver Fatigue Detection Based on Eye Tracking. In Proceedings of the International Conference on Emerging Trends in Engineering \& Technology, Nagpur, India, 16-18 July 2008.

11. Zhang, Z.; Zhang, J. Driver Fatigue Detection Based Intelligent Vehicle Control. In Proceedings of the International Conference on Pattern Recognition, Hong Kong, China, 20-24 August 2006.

12. Wang, R.; Guo, K.; Shi, S.; Chu, J. A monitoring method of driver fatigue behavior based on machine vision. In Proceedings of the Intelligent Vehicles Symposium, Columbus, OH, USA, 9-11 June 2003.

13. Mandal, B.; Li, L.; Wang, G.S.; Lin, J. Towards Detection of Bus Driver Fatigue Based on Robust Visual Analysis of Eye State. IEEE Trans. Intell. Transp. Syst. 2017, 18, 545-557. [CrossRef]

14. Saradadevi, M.; Bajaj, P. Driver Fatigue Detection Using Mouth and Yawning Analysis. Int. J. Comput. Sci. Netw. Secur. 2008, 8, 183-188.

15. Ji, Q.; Zhu, Z.; Lan, P. Real-time nonintrusive monitoring and prediction of driver fatigue. IEEE Trans. Veh. Technol. 2004, 53, 1052-1068. [CrossRef]

16. Park, S.; Pan, F.; Kang, S.; Yoo, C.D. Driver drowsiness detection system based on feature representation learning using various deep networks. In Asian Conference on Computer Vision; Springer: Cham, Switzerland, 2016; pp. 154-164.

17. Yu, J.; Park, S.; Lee, S.; Jeon, M. Representation learning, scene understanding, and feature fusion for drowsiness detection. In Asian Conference on Computer Vision; Springer: Cham, Switzerland, 2016; pp. 165-177.

18. García-García, M.; Caplier, A.; Rombaut, M. Sleep Deprivation Detection for Real-Time Driver Monitoring Using Deep Learning. In International Conference Image Analysis and Recognition; Springer: Cham, Switzerland, 2018; pp. 435-442.

19. Celona, L.; Mammana, L.; Bianco, S.; Schettini, R. A Multi-Task CNN Framework for Driver Face Monitoring. In Proceedings of the 2018 IEEE 8th International Conference on Consumer Electronics-Berlin (ICCE-Berlin), Berlin, Germany, 2-5 September 2018; pp. 1-4. 
20. Lyu, J.; Yuan, Z.; Chen, D. Long-term multi-granularity deep framework for driver drowsiness detection. arXiv 2018, arXiv:1801.02325.

21. Hochreiter, S.; Schmidhuber, J. Long short-term memory. Neural Comput. 1997, 9, 1735-1780. [CrossRef]

22. Jabbar, R.; Al-Khalifa, K.; Kharbeche, M.; Alhajyaseen, W.; Jafari, M.; Jiang, S. Real-time Driver Drowsiness Detection for Android Application Using Deep Neural Networks Techniques. Procedia Comput. Sci. 2018, 130, 400-407. [CrossRef]

23. Liu, W.; Sun, H.; Shen, W. Driver fatigue detection through pupil detection and yawning analysis. In Proceedings of the 2010 International Conference on Bioinformatics and Biomedical Technology, Chengdu, China, 16-18 April 2010.

24. Reddy, B.; Kim, Y.H.; Yun, S.; Seo, C.; Jang, J. Real-Time Driver Drowsiness Detection for Embedded System Using Model Compression of Deep Neural Networks. In Proceedings of the Computer Vision and Pattern Recognition Workshops, Honolulu, HI, USA, 21-26 July 2017; pp. 438-445.

25. Simonyan, K.; Zisserman, A. Two-stream convolutional networks for action recognition in videos. In Proceedings of the Advances in Neural Information Processing Systems 27 (NIPS 2014), Montreal, QC, Canada, 8-13 December 2014; pp. 568-576.

26. Weng, C.H.; Lai, Y.H.; Lai, S.H. Driver drowsiness detection via a hierarchical temporal deep belief network. In Proceedings of the Asian Conference on Computer Vision Workshop on Driver Drowsiness from Video, Taipei, Taiwan, 20-24 November 2016; pp. 117-133.

27. Viola, P.; Jones, M. Robust Real-time Face Detection. Int. J. Comput. Vis. 2004, 57, 137-154. [CrossRef]

28. Zhang, K.; Zhang, Z.; Li, Z.; Qiao, Y. Joint Face Detection and Alignment Using Multitask Cascaded Convolutional Networks. IEEE Signal Process. Lett. 2016, 23, 1499-1503. [CrossRef]

29. Girshick, R.; Donahue, J.; Darrell, T.; Malik, J. Rich feature hierarchies for accurate object detection and semantic segmentation. In Proceedings of the IEEE Conference on Computer Vision and Pattern Recognition, Portland, OR, USA, 23-28 June 2014; pp. 580-587.

30. Hoang, T.; Pan, B.; Nguyen, D.; Wang, Z. Generic gamma correction for accuracy enhancement in fringe-projection profilometry. Opt. Lett. 2010, 35, 1992. [CrossRef] [PubMed]

31. Farnebäck, G. Two-Frame Motion Estimation Based on Polynomial Expansion. In Proceedings of the 13th Scandinavian conference on Image analysis, Halmstad, Sweden, 29 June-2 July 2003; pp. 363-370.

32. Nayak, D.R.; Dash, R.; Majhi, B. Classification of brain MR images using discrete wavelet transform and random forests. In Proceedings of the Computer Vision, Pattern Recognition, Image Processing \& Graphics, Patna, India, 16-19 December 2016.

33. Fix, E.; Hodges, J.L., Jr. Discriminatory Analysis-Nonparametric Discrimination: Consistency Properties; California University at Berkeley: Berkeley, CA, USA, 1951.

34. Nguyen, B.P.; Tay, W.L.; Chui, C.K. Robust biometric recognition from palm depth images for gloved hands. IEEE Trans. Hum. Mach. Syst. 2015, 45, 799-804. [CrossRef]

35. Chang, C.C.; Lin, C.J. LIBSVM: A library for support vector machines. ACM Trans. Intell. Syst. Technol. (TIST) 2011, 2, 27. [CrossRef]

36. Breiman, L. Random forests. Mach. Learn. 2001, 45, 5-32. [CrossRef]

(C) 2019 by the authors. Licensee MDPI, Basel, Switzerland. This article is an open access article distributed under the terms and conditions of the Creative Commons Attribution (CC BY) license (http://creativecommons.org/licenses/by/4.0/). 